\title{
Analysis of surface temperature characteristics of multiscale fusion based on convolution neural network
}

\author{
Yixue Meng ${ }^{1, *}$ \\ ${ }^{1}$ School of geodesy and geomatics, Wuhan University, 430072 Wuhan, China
}

\begin{abstract}
Intelligent detection of surface temperature 1, describing macro characteristics of microcosmic combination, promotes the cross fusion of geoscience, thermodynamics, climatology, geological science, and so on. However, there are still two notable problems to be solved. One is the model lacks characterization capability, and the other is that the precision of surface temperature's monitoring and prediction is low. To solve these problems, we propose an algorithm to predict surface temperature characteristics of multiscale fusion based on convolution neural network. Firstly, after researching the multiscale disturbance characteristics of surface temperature, we draw a conclusion based on analyzing time change, spatial change, casual change. To improve the parameter correlations among surface temperature characteristics, a neural network about compensating and optimizing analysis of surface temperature characteristics is proposed on the fundamental of multivariate surface temperature characterization models. By designing cluster input layer, dynamic hidden layer and visual output layer of neural network, the precise of predict data has been improved by $53.3 \%$ on average, and $76.0 \%$ on variance compared with remote sensing data. What's more, the data consumption of this model has promoted by $17.2 \%$ in contrast to grey theory on predictive complexity and precision, and $10.8 \%$ compared with BP neural network.
\end{abstract}

\section{Introduction}

Intelligent detection of surface temperature, enabling human to describe macro characteristics of microcosmic combination, is a prediction on the intensity of combination molecular heat movement level about complex and large-scale land surface and heterologous structural material.

Detecting surface temperature intelligently needs multidisciplinary integration, supporting the development of geoscience, thermodynamics, climatology and geological science, which in turn promotes many cuttingedge technologies including surface temperature modeling, multivariate sensor fusion detection, surface temperature data analysis [4] and surface feature change prediction [5].

Nowadays, world powers highly valued intelligent detection of surface temperature. America investigates about 10 billion in developing earth exploration program, aiming at analyzing geological characteristics including surface temperature detected by comprehensive measures. In 2017, NASA released earth's surface temperature monitoring reports continuously, pointing out that climate, agriculture, transportation and many other factors would be influenced by surface temperature change. On the other hand, European Space Agency planned to spend 2 billion EUR on ERS (European Remote Sensing Satellite), observing surface and atmosphere continuously. Japan Space Development Corporation researched and developed ADEOS to monitor global changes of the Earth's environment.

The market scale will reach to hundreds of billions profit about intelligent detection field by 2020, which takes cores as Remote sensing satellites, surface sensors, temperature monitoring platforms and surface temperature big data services. In the meantime, indirect economic loss caused by flood and draught will be reduced considerably, by predicting surface temperature precisely.

Many other research institutes also do lots of researches on intelligent detection of surface temperature, including multi-region solar radiation [6], land surface temperature conversion rate of land use change prediction, damage analysis based on surface temperature of gravity dam [7], lake basin and surface temperature [8], the relationship between regional precipitation and surface temperature and so forth. Facing problems hard to solve like heterogeneous sensors, uncertainties in measurement factors, complex environmental changes and large-scale prediction [9] difficulties, many models are promoted such as NIVC-BP, grey theory, neural network, geographic space-time database, data mining, heterogeneous signal source fusion, which improves the efficient and precision of surface temperature monitor.

In conclusion, there are two problems to be solved under present researches of intelligent detection of surface temperature.

(1) Complex surface temperature correlation characteristics cannot be described by limited data model. 
It is difficult to induction a high universal model because its large coverage, complex factors and involving numerous data.

(2) Predicting precisely depends on sufficient prior data and expected network. Prior data includes remote sensing images and massive historical observation data, which may be inadequate for some fewer people area. It will take a lot of time for machine training for predicting surface temperature needs more parameters and big amount of data. Besides, due to the difficulty of transform non-independent space-time relationship, we need to figure out how to optimize input and output network.

We propose a predicting algorithm based on multiscale fusion of surface temperature characteristics about convolution neural network, to solve the problem of insufficient model characterization and poor accuracy in temperature monitoring. First of all, we analyze the multiscale disturbance characteristics of surface temperature, and come to conclusion about surface temperature characteristics perturbation factor analysis based on time changes, spatial changes and casual changes. In order to improve the correlation of predicting surface temperature parameters, the convolution neural network is proposed to compensate and optimize the surface temperature characteristics based on the constructed multi-surface temperature characteristic model. By designing convolutional neural network clustering input layer, dynamic hidden layer, visual output layer, we effectively enhance the accuracy of surface temperature prediction. Simulation results show that better performance in prediction complexity and precision will be achieved by the proposed algorithm.

\section{Multi-scale disturbance characteristics analysis of surface temperature}

\subsection{Distortion analysis of time varying surface temperature}

Surface temperature changes is the combined effect of macro and micro factors, which are constricted by time change, special change and casual changes. These factors, in turn, form different disturbance characteristics. Surface temperature is influenced greatly by solar radiation, precipitation, vegetation cover, water, underlying surface.

Therefore, starting from basic determining surface temperature, we correct surface temperature models based on specific perturbation characteristics. The equation of temperature measured by the infrared radiometer is

$M(T)=\varepsilon B\left(T_{s}\right)+(1-\varepsilon) E\left(T_{e}\right)$

$M(T)$ means the surface radiation received by the sensor $(\mathrm{W} /(\mathrm{m} 2 * \mathrm{um}))$, which is the integrated temperature value caused by a variety of heat through different radiation and attenuation. $T$ means surface radiation temperature $(\mathrm{K})$, which is a vital factor reflecting surface energy loss. The higher temperature is, the more energy loss there is. $\varepsilon$ means surface emissivity, which is the symbol of ground radiation flux density relating surface underlying geology. $B\left(T_{s}\right)$ means Planck's function $(\mathrm{W} /(\mathrm{m} 2 * \mathrm{um})) . T_{s}$ means the real surface temperature $(\mathrm{K})$, which is the measured temperature after a combination of various factors. $E\left(T_{e}\right)$ means the ambient irradiance $(\mathrm{W} /(\mathrm{m} 2 * \mathrm{um}))$, which is related to surface obstacles.

Substituting Stephen's-Boltzmann's law into equation (1), and we get:

$$
\sigma T^{4}=\varepsilon \sigma T_{s}^{4}+(1-\varepsilon) R_{l d}
$$

$\sigma$ means Stefan - Boltzmann constant. And after transforming equation (2), we will get the equation of real surface temperature:

$$
T_{S}=\sqrt[4]{\frac{\sigma T^{4}+(1-\varepsilon) R_{l d}}{\varepsilon \sigma}}
$$

$R_{l d}$ means sky environment radiation. The surface temperature change has a strong relationship with time, meanwhile, surface radiation temperature $T$ and surface emissivity $\varepsilon$ also vary with time. Time scale includes the day and night time difference $t_{1}$ and season difference $t_{2}$, so we have an equation:

$$
T\left(t_{1}\right)=\alpha T\left(t_{1}\right),\left\{\begin{array}{c}
\alpha<1,0<t_{1}<9 \\
\alpha>1,9<t_{1}<15 \\
\alpha<1,16<t_{1}<24
\end{array}\right.
$$

$\alpha$ means small-scale time-varying temperature difference control factor. Set 0 for the first day of the year as $t_{0}$, with 24 hours a day, 360 days a year. Since season difference is a large-scale variable, we set $\mathrm{m}=24 \times 30$ hours, and deduced the equation of surface emissivity $\varepsilon$ :

$$
\varepsilon\left(t_{2}\right)=\beta T\left(t_{2}\right),\left\{\begin{array}{c}
\beta<1,0<t_{2}<3 m \\
\beta>1,3 m<t_{2}<9 m \\
\beta<1,9 m<t_{2}<12 m
\end{array}\right.
$$

$\beta$ means large-scale time-varying temperature difference control factor.

\subsection{Spatial variation of the surface temperature characteristics of disturbance analysis}

Considering that the Earth's revolution has the greatest impact on the solar radiation reaching ground and that longitude's influence on surface temperature, we can build a model based on latitude's impact on surface temperature change:

$$
T\left(t_{1}, \varphi\right)=\gamma T\left(t_{1}, \varphi\right),\left\{\begin{array}{l}
\gamma>1,0^{\circ}<\varphi<30^{\circ} \\
\gamma \approx 1,30^{\circ}<\varphi<60^{\circ} \\
\gamma<1,60^{\circ}<\varphi<90^{\circ}
\end{array}\right.
$$

$\varphi$ means geographical latitude, and $\gamma$ means spatial variation ratio coefficient. With the same size standard time conditions, surface temperature drops as latitude increases. When it is in low latitude, spatial variation ratio coefficient is bigger than 1 , which means surface temperature is higher than global average temperature. As latitude increases to middle area, it about equals to 1 , meaning that surface temperature is about the same as global average temperature. After reaching high latitude area, the coefficient is less than 1 , and we can infer that surface temperature is lower than global average temperature because of locating in cold zone. To sum up, surface temperature drops as latitude increases. 


\subsection{Causal analysis of surface temperature perturbations}

The spatial variation is largely related to underlying surface type, mainly including urban construction land, meadow woodland, lake, bare ground and so on. The capacity of absorbing solar radiation differs as underlying surface changes, and so is its reflection capacity. Therefore, spatial variation has a tremendous influence on surface temperature disturbance.

The relationship between surface temperature and land cover structure is complicated, making it hard to use thermodynamics and geography model to describe simple spatial distribution directly. So, we use existing satellite remote sensing surface temperature thermodynamic map to inverse surface temperature. Then segment, refine and relevance reason surface temperature distribution image, to derive the influence of surface temperature change from various underlying surface types.

Specific method is as followed: obtain the global surface image, and convert the brightness value of the image into the radiation temperature. Then obtain the corresponding pixel brightness temperature. Coupled with surface radiative temperature, building an approximate model of surface temperature through a single-channel algorithm. With this theory, equation (5) can be transformed as:

$$
\left\{\begin{array}{c}
\varepsilon\left(t_{2}, s\right)=0.921, s<0.157 \\
\varepsilon\left(t_{2}, s\right)=1.0094+0.047 \ln (s), 0.157 \leq s \leq 0.727 \\
\varepsilon\left(t_{2}, s\right)=0.9942, s>0,727
\end{array}\right.
$$

$\varepsilon$ means emissivity, and $s$ means NDVI (Normalized Difference Vegetation Index). From equation (7), we can infer that when $s$ is less than 0.157 , the type of underlying surface is bare soil, at this time, we set surface emissivity as 0.921 . When $s$ is between 0.157 and 0.727 , calculating surface emissivity according to Van de Griend \& Owe's empirical formula. And if $s$ is bigger than 0.727 , considering underlying surface type as vegetation, we set surface emissivity as 0.9942 . Besides, if the underlying surface type is water, surface emissivity is 0.995 .

More research shows that, as surface emissivity differs, it will influence surface temperature further.

\section{Compensation and optimization of surface temperature characteristic analysis based on convolution neural network}

Multi-scale disturbance modeling of surface temperature, analyzes disturbance factors of surface temperature characteristics base on time changes, spatial changes and casual changes. However, model refinement brings problems like predicting surface temperature involving more parameters, machine training requiring more time and change the non-independent space-time relationship.

To enhance parameter relevance of predicting surface temperature, a model to compensate and optimize surface temperature characteristics analysis based on convolution neural network, including clustering input layer design, dynamic hidden layer design and visual output design. As we can see in Figure 1.

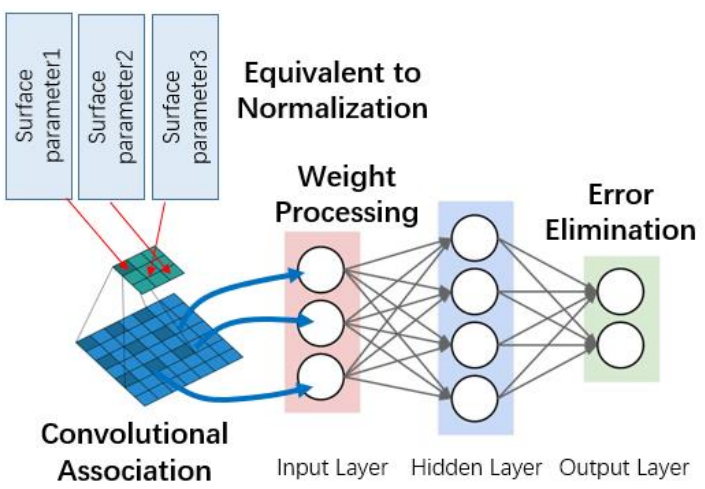

Figure 1. Convolution Neural Network for Surface Temperature Treatment

\subsection{Convolutional neural network clustering input layer design}

The basic use of neural network input layer [10]. is to process a large number of non-linear input information. The input information, called as input vector, is the original input data. We consider surface temperature changes as image pixels, and use it to compose the original input matrix, which successfully convert heterogeneous factors such as time change, spatial change and casual change into uniform input element $w$. This element $w$ is a matrix of order $i * j$, and the $j$ th output of the current $i$ th layer is $w_{j}^{i}$.

So, we firstly select several $i$ th layers from $i$ th feature group in the surface temperature influence factor matrix, and output feature set $M_{i}$. Then convolute convolution kernel $K_{i j}^{i}$ with the input characteristics of the feature set $w_{j}^{i-1}$ respectively, and calculate summation. Finally, merge with gene $b_{j}^{i}$ and we will get this equation:

$w_{j}^{i}=f\left(\sum_{i \in M_{i}} w_{j}^{i-1} K_{i j}^{i}+b_{j}^{i}\right)$

$f$ means the set processing function.

\subsection{Convolutional Neural Network Dynamic Hidden Layer Design}

Hidden layer, between the various layers of neurons and links of the input layer and output layer, processes data. We need to extract hierarchical features from influencing factor matrix [11] to complete surface temperature forecast. We extract detail features of surface temperature influencing factors from shallow layer, and general principles from deep layer. Since in layer-by-layer feature extraction, the scope is expanded, which leads to hidden layer extraction results is highly abstracted. Therefore, we need to regress the results again.

Abstracting each level needs to reprocess the weight of connecting neurons, which indeed is polynomial coefficients. Besides, the additional unit of bias is constant term, and the input and output values of the middle layer can be regarded as higher-level eigenvectors $X$. Whereas, the resulting error is the object which needs to be 
processed by hidden layer. Therefore, the linear regression error vector can be expressed as:

$$
\begin{gathered}
x=\left[\begin{array}{l}
x_{0} \\
x_{1} \\
x_{2} \\
x_{3}
\end{array}\right] \begin{array}{c}
z^{(2)}=\theta^{(1)} x \\
z^{(2)}=g\left(z^{(2)}\right) \\
h \theta(x)=\theta^{(2)} a^{(2)}=g\left(z^{(3)}\right)
\end{array}
\end{gathered}
$$

$Z^{(n)}$ means different orders of transmission error, and $\theta^{(n)}$ means the implied factor of weight.

\subsection{Convolutional Neural Network Dynamic Hidden Layer Design}

Output layer transfer, analyze, trade off, and form output results in neural network. The output information is called as output vector. The influencing factors which has been processed by input layer and hidden layer, will fit and forecast surface temperature in the output layer. Finally, the results are outputted. Among them, whether the model is accurate and can be used widely is the key to forecast result precisely.

To improve the output accuracy [12] of surface temperature, neural network is trained by using output data as the next level of neural network input data to reason again. By applying the principle of error back propagation, the network weights are constantly adjusted so that the sum of squared errors between the output of the network model and the known output value of training samples reaches the minimum or less than a certain expected value.

\section{Simulation and analysis}

\subsection{Build experimental environment}

On the basis of compensate and optimize the analysis of surface temperature characteristic, we build a more precise mathematic model learnt from multi-scale disturbance modeling of surface temperature, by constructing convolution neural network. To better verify performance, further simulation will be done to test the improvement of prediction complexity and accuracy. Experimental environment is shown as Figure 2.

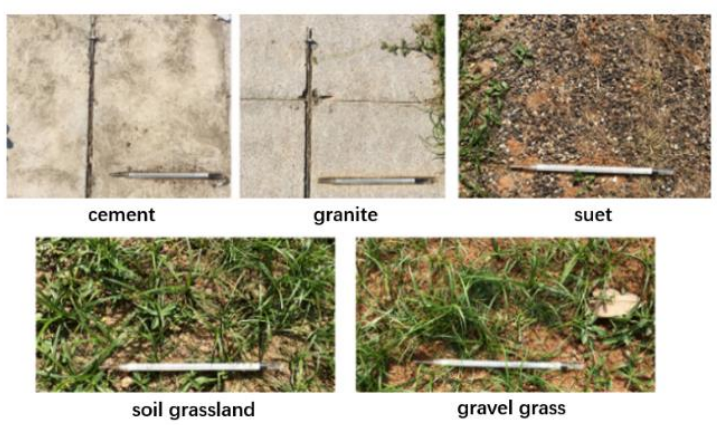

Figure 2. the actual scene of the experiment

Considering that the amount of global surface temperature data is huge, the difficulty of obtaining original data is high, and the period of analyzing and processing is long, we use simulation analysis to carry out simulation experiment and precision analysis in local area, which will verify the accuracy of the model. We choose ground and shallow surface temperature as experiment zone, setting at flat bare land in the south of the Wuhan University campus garden. The area is $2 \times 4 \mathrm{~m}^{2}$, with loose, smooth, grass-free surface.

\subsection{Contrast of analysis precision}

Analysis accuracy is an important index to measure the difference between surface temperature model and real surface temperature. Under original condition or simulation condition, model accuracy can be analyzed from same period and different period. Furthermore, another important index to measure model accuracy is using the model to get precise surface temperature in the future.

Table 1. Comparison of Surface Temperature Accuracy of Different Models with Different Underlying Surfaces

\begin{tabular}{|c|c|c|c|}
\hline $\begin{array}{c}\text { Surface/Period } \\
\left({ }^{\circ} \mathrm{C}\right)\end{array}$ & $\begin{array}{c}\text { Actual } \\
\text { measurement } \\
\text { value }\end{array}$ & $\begin{array}{c}\text { Remote } \\
\text { sensing } \\
\text { value }\end{array}$ & $\begin{array}{c}\text { Model } \\
\text { prediction } \\
\text { value }\end{array}$ \\
\hline Soil grassland & 18.2 & 18.4 & 18.3 \\
\hline Cement & 15.3 & 15.6 & 15.4 \\
\hline Gravel grass & 19.1 & 19.3 & 19.2 \\
\hline Suet & 18.6 & 18.4 & 18.5 \\
\hline Granite & 16.7 & 16.5 & 16.6 \\
\hline
\end{tabular}

We can infer from Table 1, the difference of actual measurement value and remote sensing value is -0.06 on average, and 0.046 on variance. However, compared with model prediction, the difference is -0.028 on average and 0.011 on variance. We can calculate from these data that if we use this model to predict surface temperature, the accuracy will improve by $53.3 \%$ on average, and $76.0 \%$ on variance. It is clearly to see that this model is more reliable to calculate surface temperature than remote sensing data, under different circumstances where a variety of underlying surface type and time.

\subsection{Contrast of analysis complexity}

Analysis complexity is measured by the resources and operational efficiency which is needed when operating this model. The reason why analysis complexity is important is that the original data of surface temperature model is numerous, the involving parameters are a lot and they are relevant and no change in law. Therefore, we need to test whether the model is efficient, accurate and wildly used by analyzing complexity.

We simulate and transform local area data, then compare them with known model to test its processing time, efficiency and accuracy. 
Table 2 Different average difference consumption calculated by different methods

\begin{tabular}{|c|c|c|c|}
\hline $\begin{array}{c}\text { Measureme } \\
\text { nt object } \\
\text { data volume }\end{array}$ & $\begin{array}{c}\text { Gray } \\
\text { theoretica } \\
1 \text { data } \\
\text { volume }\end{array}$ & $\begin{array}{c}\text { BP } \\
\text { neural } \\
\text { networ } \\
\text { k data } \\
\text { volume }\end{array}$ & $\begin{array}{l}\text { Model } \\
\text { reasonin } \\
\text { g data } \\
\text { volume }\end{array}$ \\
\hline $\begin{array}{c}\text { average } \\
\text { difference } \\
10 \%\end{array}$ & $136 \mathrm{MB}$ & $129 \mathrm{MB}$ & $112 \mathrm{MB}$ \\
\hline $\begin{array}{c}\text { average } \\
\text { difference } \\
5 \%\end{array}$ & $239 \mathrm{MB}$ & $222 \mathrm{MB}$ & $198 \mathrm{MB}$ \\
\hline $\begin{array}{c}\text { average } \\
\text { difference } \\
2 \%\end{array}$ & $556 \mathrm{MB}$ & $543 \mathrm{MB}$ & $387 \mathrm{MB}$ \\
\hline
\end{tabular}

As we can see in table 2, when the average difference is $10 \%$, the grey theory data consumption is $136 \mathrm{MB}$, and BP neural network data consumption is $129 \mathrm{MB}$. So, the model reasoning data consumption is improved $17.6 \%$ compared with grey theory, and $13.2 \%$ compared with BP neural network. When the average difference is $5 \%$, the grey theory data consumption is $239 \mathrm{MB}$, and $\mathrm{BP}$ neural network data consumption is $222 \mathrm{MB}$. The model reasoning data consumption is improved $17.2 \%$ compared with grey theory, and $10.8 \%$ compared with BP neural network. When the average difference is $2 \%$, the grey theory data consumption is $556 \mathrm{MB}$, and $\mathrm{BP}$ neural network data consumption is $543 \mathrm{MB}$. So, the model reasoning data consumption is improved $30.4 \%$ compared with grey theory, and $28.7 \%$ compared with BP neural network. Therefore, in contrast with the former two models, this model reasoning result has fewer data consumption. Besides, the litterer average difference is, the more obvious enhancement effect is.

\section{Conclusion}

We propose an algorithm to predict multiscale fusion of surface temperature characteristics base on convolution neural network, which is on the fundamental of surface temperature characteristics perturbation factor mathematical model relating to time change, spatial change and casual change. This algorithm has solved the problem that the influence of surface temperature is diversified. Then, on the basis of convolution neural network, we realize clustering input layer design, dynamic hidden layer design and visual output design, dealing with the problem that surface temperature parameters are highly correlated. This design makes the accuracy of predicted date improved by $53.3 \%$ on average and $76.0 \%$ on variance, compared with remote sensing data. In the last, we carry out simulation experiment for this model, proving that the predict complexity and accuracy of data consumption has been improved by $17.2 \%$ compared with the grey theory, and $10.8 \%$ compared with BP neural network.

\section{References}

1. Palczewski, A., Geng, R., \& Eremeev, G. (2011). Design, construction, and initial test of high spatial resolution thermometry arrays for detection of surface temperature profiles on srf cavities in super fluid helium. Office of Scientific \& Technical Information Technical Reports.

2. Chen, Y., Yang, K., Jie, H., Qin, J., Shi, J., \& Du, J., et al. (2011). Improving land surface temperature modeling for dry land of china. Journal of Geophysical Research Atmospheres, 116(D20)

3. Bahrepour, M., Meratnia, N., \& Havinga, P. J. M. (2009). Sensor fusion-based event detection in Wireless Sensor Networks. Mobile and Ubiquitous Systems: NETWORKING \& Services, Mobiquitous, 2009. MOBIQUITOUS '09. International (pp.1-8). IEEE.

4. Sedighi, M., Bennett, D. P., Masum, S. A., Thomas, H. R., Johansson, E., \& Siren, T. (2014). Temperature data analysis from field scale thermal monitoring. Human rights in the twenty-first century :. M. Nijhoff.

5. Zhao, Y., \& Chen, X. (2011). Prediction-based geometric feature extraction for $2 \mathrm{~d}$ laser scanner. Robotics \& Autonomous Systems, 59(6), 402-409.

6. Guermoui, M., Rabehi, A., Benkaciali, S., \& Djafer, D. (2016). Daily global solar radiation modelling using multi-layer perceptron neural networks in semiarid region. , 15(28).

7. Fan, S. L., Chen, M. Y., Chen, J. Y., \& Chai, H. C. (2011). Seismic damage analysis of a concrete gravity dam based on energy dissipation. Journal of Vibration \& Shock, 30(4), 271-275.

8. Sun, X., Xie, L., Semazzi, F., \& Liu, B. (2013). Effect of lake surface temperature on the spatial distribution and intensity of the precipitation over the lake victoria basin. Monthly Weather Review, 143(4), 1179-1192.

9. Tian, H., Xu, X., Zhang, C., Ren, W., Chen, G., \& Liu, M., et al. (2015). Forecasting and assessing the largescale and long-term impacts of global environmental change on terrestrial ecosystems in the united states and china. Research Technology Management, volume 51, 18-22(5).

10. Sun, Y., Zhang, N., Lu, X., Yang, H., \& Yue, Z. (2010). Power consumption prediction of submerged arc furnace based on multi-input layer wavelet neural network. International Conference on Mechanic Automation and Control Engineering (pp.3586-3589). IEEE.

11. Jian, M. U., Liu, S. Y., \& Xin-Yan, X. U. (2013). A study of power grid planning management mechanism based on influencing factors matrix method. Journal of Electric Powe

12. Techcon, A. (2014). Hands on: improve the output accuracy over temperature for rms power detectors. Eetimes Com. r. 